\title{
An integrated project for teaching the post-buckling of a slender cantilever bar
}

T. Beléndez (1), C. Neipp (2) and A. Beléndez (2)

(1) Departamento de Ciencia y Tecnología de los Materiales. Universidad Miguel Hernández de Elche.

Avda. del Ferrocarril, s/n. E-03202. Elche (Alicante). SPAIN

(2) Departamento de Física, Ingeniería de Sistemas y Teoría de la Señal. Universidad de Alicante. Apartado 99. E-03080 Alicante. SPAIN

Corresponding author: A. Beléndez

Phone: +34-6-5903651

Fax: +34-6-5903464

E-mail: augusto@disc.ua.es

\section{ABSTRACT}


We describe an integrated project that includes theoretical, numerical and experimental studies designed to facilitate teaching the non-linear post-buckling of a cantilever column to undergraduate mechanical engineering students. We present the differential equation governing the behaviour of this mechanical system and show that this equation, although straightforward in appearance, is in fact rather difficult to solve due to the presence of a non-linear term. In this sense, this system is similar to another well known physical system: the simple pendulum motion. This educational paper also addresses a simplified procedure for calculating the elliptical integrals that appear when the differential equation is solved. We present numerical results for various cases. Finally, we compare the theoretical results with the experimental ones obtained in the laboratory using materials which are easily and cheaply sourced.

KEYWORDS: Cantilever column, post-buckling, geometrical nonlinearity. 


\section{1.- Introduction}

In mechanics there are some simple systems for which the equations governing their behaviour are easy to formulate but whose mathematical resolution is complicated. Of all such systems, perhaps the paradigm that is usually considered in physics courses at university level is the simple pendulum [1-4]. Application of Newton's second law to this physical system gives a differential equation with a nonlinear term (the sine of an angle). It is possible to find the integral expression for the period of the pendulum from the law of conservation of energy and to express this period in terms of elliptic functions. However, if we consider the case of small oscillations it is possible to substitute the sine of the angle by the angle itself, thereby obtaining a second order linear differential equation with constant coefficients, the solution of which is the well-known harmonic function, and an amplitude-free period is obtained [5].

We shall analyze another example of a simple mechanical system, the postbuckling of a slender cantilever bar. We shall see that it is not complicated to formulate the equations governing its behaviour or to study this system in a mechanics laboratory at university level. However, a differential equation with a nonlinear term is once again obtained. The problem is said to involve geometric nonlinearity. Moreover -as occurs with the simple pendulum for small oscillations- when small deflections of the cantilever bar are considered, it is possible to find a simple analytical solution to the problem. In this sense, the study of the post-buckling of a slender cantilever slender bar shows analogies with the study of the oscillations of a simple pendulum.

Cantilever bars and columns are common elements of many architectural, civil and mechanical engineering structures. In the laboratory it is possible to design simple 
experiments in order to analyze the post-buckling of a slender cantilever bar with a tip load applied at the end free. For example, Figure 1 shows a photograph of a system made up of a steel ruler of rectangular section built-in at one end and loaded at the free end with a mass. The ruler is fixed by means of a multi-clamp using two small metallic pieces, which provide a better support. With this experimental set-up the students can, for instance, determine the horizontal and vertical deflections at the free end as a function of the applied load, or the shape the ruler adopts under the action of that force, and they can relate these measurements to geometric parameters of the bar (its length and the inertial moment of its rectangular cross-section), as well as to the material of which it is made (using Young's modulus). As can be seen in Figure 1, it is a physical system which is easy to assemble and analyze in the physics laboratory in first year university courses. This system is also composed of very simple elements and it is only necessary to make easy experimental measurements (basically lengths and masses). In addition, mathematical treatment of the equilibrium of the system does not involve great difficulty [6]. Nevertheless, unless small deflections are considered, an analytical solution does not exist, since for large deflections a differential equation with a non-linear term must be solved.

The purpose of our study is to analyze the classical problem of the postbuckling of a slender cantilever column of linear elastic material, under the action of an external vertical concentrated load at the free end. Under the action of this external force, the column deflects into a curve. This is the elastica problem, which was originally solved by Leonard Euler (1707-1783) and published in the appendix, "De curvis elasticis" of his book "Methodus inveniendi lineas curvas maximi minimive proprietate gaudentes" [7-9]. 
The treatment of the problem of post-buckling of a slender bar can be found in university textbooks on physics, mechanics and elementary mechanics of materials. However, in these books the discussion is limited to the consideration of small deflections and they present a formula for the critical load (or Euler load), that is the smallest axial force which can maintain the bar in a slightly bent shape. The critcal load can be easily calculated using the differential equation for the deflection curve for small deflections. As we show in this paper, the problem is similar to the analysis of the motion of a simple pendulum for a small initial amplitude. The analysis of large deflections of these types of cantilever bars of elastic material can be found in Landau's book on elasticity [10], and the solution in terms of elliptic functions can be found in specialized books on non-linear mechanics [11]. Nevertheless, the developments presented in these last references are difficult for first year university students.

In this paper we analyze the problem of the post-buckling of a slender, ideal bar built in vertically at the base, free at the upper end and subjected to a vertical concentrated load at the free end. Firstly, we present the differential equation for the deflection curve (elastic curve) in the general case of large deflections, as well as the equations that determine the Cartesian coordinates of each point on the elastic curve. For each one of these points we define a set of non-dimensional coordinates and a non-dimensional load parameter, which allow the solution to be expressed in a more general way. These equations will be solved numerically in an easy way with the aid of symbolic algebra software, such as Mathematica, on a personal computer, without using elliptic functions. Students on mechanics of materials courses may benefit from this alternative computational procedure. Various general numerical results are 
presented and, by way of example, these are compared with the experimental results obtained in the laboratory.

\section{2.- Theoretical analysis}

We consider a long, slender, cantilever bar of uniform cross section. The bar is assumed to be perfectly elastic, and the stresses do not exceed the proportional limit. The law of behaviour of the material (stress-strain relation) is represented by the linear relation $[6]$ :

$$
\sigma=\varepsilon E
$$

known as Hooke's law, where $\sigma$ is the normal stress (the force per unit area on a surface element within the beam, $\sigma=\mathrm{d} F / \mathrm{d} S$ ), $\varepsilon$ is the strain (the change in length of an element divided by its original length, $\varepsilon=\Delta l / l$ ) and $E$ is the modulus of elasticity or Young's modulus. In this study, we assume that the beam is non-extensible and strains remain small. Firstly, we assume that Bernoulli-Euler's hypothesis is valid, i.e., plane cross-sections which are perpendicular to the neutral axis before deformation remain plane and perpendicular to the neutral axis after deformation. Next, we also assume that the plane-sections do not change their shape or area.

Following, for instance, the analysis proposed by Feynman regarding the study of the post-buckling of a slender bar, it is possible to write the Bernoulli-Euler bending moment-curvature relationship as follows [6]:

$$
M=\frac{E I}{\rho}
$$


Where $M$ and $\rho$ are the bending moment and the curvature radius at any point of the bar, respectively, and $I$ is the moment of inertia (the second moment of area) of the beam cross-section. The product EI, which depends on the type of material and the geometrical characteristics of the cross-section of the beam, is known as the flexural rigidity. We also consider that the flexural rigidity is constant. Taking into account the relation $1 / \rho=\mathrm{d} \varphi / \mathrm{d} s$, where the definition of $\varphi$ and $s$ can be seen in Figure 2, it is possible to write equation (2) as follows:

$$
M=E I \frac{\mathrm{d} \varphi}{\mathrm{d} s}
$$

This equation -which involves the bending moment, $M$-governs the deflections of beams and bars made of linear type material under general loading conditions.

We will consider the post-buckling of a slender cantilever bar, subjected to one vertical concentrated load at the free end, by supposing that the deflection due to its own weight is null. This implies considering a massless bar. As we will see this approximation is valid if the bar's own weight is much less than the critical load.

Figure 2 shows a vertical slender cantilever bar of length $L$ with a concentrated load $F$ applied at the free end of the bar. In this figure $x_{f}$ and $y_{f}$ are the vertical and horizontal displacements at the free end, respectively, and $\varphi_{0}$ takes into account the slope of the bar at the free end. We take the origin of the Cartesian coordinate system at the fixed end of the bar and let $(x, y)$ be the coordinates of point $A$, and $s$ the arc length of the bar between the fixed end and point $A$. In order to obtain the equation that governs the post-buckling of the bar, we differentiate equation (3) once with respect to $s$, and we obtain: 


$$
E I \frac{\mathrm{d}^{2} \varphi}{\mathrm{d} s^{2}}=\frac{\mathrm{d} M}{\mathrm{~d} s}
$$

In this equation we assume constant lengthwise cross-sectional area and moment of inertia. The differential equation (4) involves the shearing force $\mathrm{d} M / \mathrm{d} s$ instead of the bending moment $M$, as appeared in equation (3). The bending moment $M$ at a point $A$ with Cartesian coordinates $(x, y)$ is given by the equation:

$$
M(s)=F\left(x_{f}-x\right)
$$

where $x_{f}-x$ is the distance along the $x$-axis from the section of the bar at a point $A$ to the free end where force $F$ is applied (Figure 2).

By differentiating equation (5) once with respect to $s$, we obtain:

$$
\frac{\mathrm{d} M}{\mathrm{~d} s}=-F \sin \varphi
$$

where the relation $\sin \varphi=\mathrm{d} x / \mathrm{d} s$ has been taken into account (Figure 2). Substituting equation (6) in equation (4), we obtain the non-linear differential equation that governs the post-buckling of a slender cantilever bar made of a linear material under the action of a vertical concentrated load at the free end:

$$
E I \frac{\mathrm{d}^{2} \varphi}{\mathrm{d} s^{2}}+F \sin \varphi=0
$$


In this equation $\varphi(s)$ is the slope at any point along the arc length with respect to the horizontal, and $s$ is the arc length measured from the fixed end. Equation (7), although straightforward in appearance, is in fact rather difficult to solve because of the non-linearity inherent in the term $\sin \varphi$. As indicated in the introduction, this situation is similar to that which can be found in the study of the motion of a simple pendulum. The movement equation of a simple pendulum is similar to equation (7) (though for a simple pendulum of length $l$ in a (uniform) gravitational field $g$, this equation depends on the time $t$ instead of the arc length $s$, and $l / g$ appears instead of $F / E I)$ and for small oscillations only it is possible to obtain an easy analytical solution for the movement of the pendulum.

In order to obtain the solution of equation (7), this equation is multiplied by $\mathrm{d} \varphi / \mathrm{d} s$, so that it becomes:

$$
E I \frac{\mathrm{d} \varphi}{\mathrm{d} s} \frac{\mathrm{d}^{2} \varphi}{\mathrm{d} s^{2}}+F \sin \varphi \frac{\mathrm{d} \varphi}{\mathrm{d} s}=0
$$

which can be written as:

$$
\frac{\mathrm{d}}{\mathrm{d} s}\left[\frac{1}{2} E I\left(\frac{\mathrm{d} \varphi}{\mathrm{d} s}\right)^{2}-F \cos \varphi\right]=0
$$

where we have taken into account the relation:

$$
\frac{\mathrm{d}^{2} \varphi}{\mathrm{d} s^{2}}=\frac{1}{2} \frac{\mathrm{d}}{\mathrm{d} s}\left(\frac{\mathrm{d} \varphi}{\mathrm{d} s}\right)^{2}
$$

Equation (9) is immediately integrable and it is possible to obtain the following expression: 


$$
\frac{1}{2} E I\left(\frac{\mathrm{d} \varphi}{\mathrm{d} s}\right)^{2}-F \cos \varphi=c_{1}
$$

where $c_{1}$ is an integration constant. To obtain $c_{1}$ we take into account that at the free end $\varphi(L)=\varphi_{0}$, where $\varphi_{0}$ is the unknown slope at the free end of the bar (see Figure 3), and from equations (3) and (5), it follows that:

$$
\left(\frac{\mathrm{d} \varphi}{\mathrm{d} s}\right)_{s=L}=0
$$

Then, from equations (11) and (12) we obtain $c_{1}=-F \cos \varphi_{0}$, and equation (11) can be written as:

$$
\left(\frac{\mathrm{d} \varphi}{\mathrm{d} s}\right)^{2}=\frac{2}{E I}\left(F \cos \varphi-F \cos \varphi_{0}\right)
$$

and by integrating it we can obtain the following equation for the arc length $s$ as a function of the slope $\varphi$ of any point along the arc length with respect to the $x$-axis:

$$
s=\sqrt{\frac{E I}{2 F}} \int_{0}^{\varphi} \frac{\mathrm{d} \varphi}{\sqrt{\cos \varphi-\cos \varphi_{0}}}
$$

The total length $L$ corresponds to the unknown angle $\varphi_{0}$ at the free end of the beam:

$$
L=\sqrt{\frac{E I}{2 F}} \int_{0}^{\varphi_{0}} \frac{\mathrm{d} \varphi}{\sqrt{\cos \varphi-\cos \varphi_{0}}}
$$


Equation (15) allows us to obtain the angle $\varphi_{0}$ at the free end of the bar as a function of the length, $L$, the modulus of elasticity, $E$, the moment of inertia of the cross-section of the bar, $I$, and the external load, $F$. After obtaining the arc length $s$ as a function of $\varphi$ (equation (14)), and taking into account that $\sin \varphi=\mathrm{d} x / \mathrm{d} s$ and $\cos \varphi=$ $\mathrm{d} y / \mathrm{d} s$, the $x$ and $y$ coordinates of the horizontal and vertical deflection at any point along the neutral axis of the cantilever beam are found as follows:

$$
\begin{gathered}
x=\sqrt{\frac{2 E I}{F}}\left(\sqrt{1-\cos \varphi_{0}}-\sqrt{\cos \varphi-\cos \varphi_{0}}\right) \\
y=\sqrt{\frac{E I}{2 F}} \int_{0}^{\varphi} \frac{\cos \varphi \mathrm{d} \varphi}{\sqrt{\cos \varphi-\cos \varphi_{0}}}
\end{gathered}
$$

From Figure 2, it is easy to see that the horizontal and vertical displacements at the free end can be obtained from equations (16) and (17) taking $\varphi=\varphi_{0}$ :

$$
\begin{aligned}
& x_{f}=x\left(\varphi_{0}\right) \\
& y_{f}=y\left(\varphi_{0}\right)
\end{aligned}
$$

We introduce the non-dimensional load parameter $k$, which is proportional to the external load $F$ and defined as follows:

$$
k=\frac{F L^{2}}{E I}
$$

We also introduce the non-dimensional coordinates: 


$$
\begin{aligned}
& u=x / L \\
& v=y / L
\end{aligned}
$$

as well as the non-dimensional tip deflection ratios:

$$
\begin{aligned}
& u_{f}=x_{f} / L=u\left(\varphi_{0}\right) \\
& v_{f}=y_{f} / L=v\left(\varphi_{0}\right)
\end{aligned}
$$

Using the parameter $k$, it is possible to obtain a more general view of the results, because cantilever bars with different combinations of $E, I, F$ and $L$ may give the same value of $k$ and, consequently, they would have the same behavior.

Taking into account the definitions of $k, u$ and $v$, equations (15), (16) and (17) can be written as:

$$
\begin{gathered}
\int_{0}^{\varphi_{0}} \frac{\mathrm{d} \varphi}{\sqrt{\cos \varphi-\cos \varphi_{0}}}-\sqrt{2 k}=0 \\
u=\sqrt{\frac{2}{k}}\left(\sqrt{1-\cos \varphi_{0}}-\sqrt{\cos \varphi-\cos \varphi_{0}}\right) \\
v=\frac{1}{\sqrt{2 k}} \int_{0}^{\varphi} \frac{\cos \varphi \mathrm{d} \varphi}{\sqrt{\cos \varphi-\cos \varphi_{0}}}
\end{gathered}
$$

Equation (25) allows us to obtain $\varphi_{0}$ as a function of the non-dimensional load parameter $k$. However, equations (25) and (27) are elliptic integrals that may be evaluated numerically. Given the vertical external force $F$, the free-end location parameters can be determined from equations (23), (24), (26) and (27) after 
evaluation of the angle $\varphi_{0}$ in equation (25). However, equation (25) implicitly relates $\varphi_{0}$ to the load $F$ by means of $k$.

In the case of small deflections, the angle $\varphi$ is small for all the points on the cantilever beam. If $\varphi$ and $\varphi_{0}$ are sufficiently small, the approximations:

$$
\cos \varphi \approx 1-\frac{\varphi^{2}}{2} \quad \cos \varphi_{0} \approx 1-\frac{\varphi_{0}^{2}}{2}
$$

may be used in equations (25)-(27). From equation (25), we can write:

$$
\int_{0}^{\varphi_{0}} \frac{\mathrm{d} \varphi}{\sqrt{\varphi_{0}^{2}-\varphi^{2}}}-\sqrt{k}=0
$$

and integrating it, we can easily see that, for small deflections, the value of the angle at the free end of the beam, $\varphi_{0}$, disappears from the equation and we obtain that the load parameter, $\mathrm{k}$, introduced in the previous section, is equal to $\pi^{2} / 4$. Taking into account the definition of $k$ (equation (20)), $F$ will be equal to $\pi^{2} E I / 4 L^{2}$. This implies that $F$ is not dependent on the angle $\varphi_{0}$ for small deflections. When the applied force is less than $\pi^{2} E I / 4 L^{2}$, there is no deflection of the bar and hence no buckling [6]. If the applied force $F$ is gradually increased, the straight form of equilibrium becomes unstable and a small lateral force will produce a deflection which does not disappear when the lateral force is removed. The critical load (or Euler load), $F_{c r}$, is then defined as the smallest axial force which can maintain the bar in a slightly bent form, and for the slender cantilever bar we are analyzing its value is: 


$$
F_{c r}=\frac{\pi^{2} E I}{4 L^{2}}
$$

If we analyze the post-buckling of a slender cantilever bar under a combined load consisting of a uniformly distributed axial load (its own weight) and a concentrated load at the free end, we obtain a differential equation that must be solved using complex numerical integration procedures. From the specialized bibliography [12] it is possible to see that the effect of the bar's own weight, $W$, on the magnitude of $F_{c r}$ is equivalent to a load of $0.3 \mathrm{~W}$ applied at the top of the bar, and the critical load is now:

$$
F_{c r}^{\prime}=\frac{\pi^{2} E I}{4 L^{2}}-0.3 W
$$

The critical requirement for disregarding the effect of a beam's weight on its buckling and post-buckling performance is that the weight $W$ should be much less than the Euler load, $F_{c r}$. Then equations (25)-(27) can be used to study the postbuckling of a slender cantilever bar with a concentrated load at the free end.

\section{3.- Numerical calculation}

As mentioned above, in order to study the post-buckling of a cantilever bar subjected to a vertical concentrated load at the free end, it is necessary to know the angle $\varphi_{0}$. To do this, it is necessary to solve equation (5) in order to obtain $\varphi_{0}$ as a function of $k$. Equations (25) and (27) are elliptical integrals and their evaluation is too mathematically advanced for first year university students. Instead of writing equations (25) and (27) in terms of elliptic functions by means of complex changes of 
variable which give little insight as to the nature of the solutions, it is easier for the students to solve the equations numerically using one of the packages of commercial software available. Nowadays, all university students of science and engineering are familiar with software such as MathCad, Derive, Mathematica or Matlab, which also have student versions.

Since there is a singularity at $\varphi=\varphi_{0}$, the integral (25) is broken into two parts:

$$
\int_{0}^{\varphi_{0}-\varepsilon} \frac{\mathrm{d} \varphi}{\sqrt{\cos \varphi-\cos \varphi_{0}}}+\int_{\varphi_{0}-\varepsilon}^{\varphi_{0}} \frac{\mathrm{d} \varphi}{\sqrt{\cos \varphi-\cos \varphi_{0}}}-\sqrt{2 k}=0
$$

where $\varepsilon$ is a very small number. The first term in equation (32) is evaluated using the Mathematica program, with the aid of the "NIntegrate" command, for there is no singularity. The second term still has a singularity. Using transformation of the integral variable $z=\varphi_{0}-\varphi$, the second term in equation (32) can be changed to:

$$
\int_{\varphi_{0}-\varepsilon}^{\varphi_{0}} \frac{\mathrm{d} \varphi}{\sqrt{\cos \varphi-\cos \varphi_{0}}}=\int_{0}^{\varepsilon} \frac{\mathrm{d} z}{\sqrt{\cos \left(\varphi_{0}-z\right)-\cos \varphi_{0}}}
$$

We can write:

$$
\cos \left(\varphi_{0}-z\right)-\cos \varphi_{0}=\cos \varphi_{0} \cos z+\sin \varphi_{0} \sin z-\cos \varphi_{0}
$$

Since $z$ is a very small number, it is possible to make the approximations $\cos z \approx 1$ and $\sin z \approx z$ in equation (34), and equation (33) then takes the form:

$$
\int_{\varphi_{0}-\varepsilon}^{\varphi_{0}} \frac{\mathrm{d} \varphi}{\sqrt{\cos \varphi-\cos \varphi_{0}}}=\int_{0}^{\varepsilon} \frac{\mathrm{d} z}{\sqrt{z \sin \varphi_{0}}}=2 \sqrt{\frac{\varepsilon}{\sin \varphi_{0}}}
$$

and equation (32) can be approximately calculated as follows: 


$$
\int_{0}^{\varphi_{0}-\varepsilon} \frac{\mathrm{d} \varphi}{\sqrt{\cos \varphi-\cos \varphi_{0}}}+2 \sqrt{\frac{\varepsilon}{\sin \varphi_{0}}}-\sqrt{2 k}=0
$$

In order to evaluate equation (36) we take $\varepsilon=10^{-12}$ and we vary the values of $\varphi_{0}$ (the incognita). In Figure 3, the values of the function $f\left(\varphi_{0}\right)$ have been represented as a function of $\varphi_{0}$ for $k=3$, where $f$ is defined as follows:

$$
f\left(\varphi_{0}\right)=\int_{0}^{\varphi_{0}-\varepsilon} \frac{\mathrm{d} \varphi}{\sqrt{\cos \varphi-\cos \varphi_{0}}}+2 \sqrt{\frac{\varepsilon}{\sin \varphi_{0}}}-\sqrt{2 k}
$$

As can be seen, equation (36) can be written as $f\left(\varphi_{0}\right)=0$ and, when $k$ is greater than $\pi^{2} / 4$, we can see from Figure 3 that a unique solution for $\varphi_{0}$ exists. However, it is important to point out that when $k$ is less than $\pi^{2} / 4$ there is actually no solution for $f\left(\varphi_{0}\right)=0$ other than $\varphi_{0}$ equaling zero (i. e. the curve of $f\left(\varphi_{0}\right)$ versus $\varphi_{0}$ is always positive). Using the Mathematica program, we obtained the values of $\varphi_{0}$ as a function of $k$. To do this, for a curve such as that in Figure 3, the value of $\varphi_{0}$ (for each k) may be determined from the equation $f\left(\varphi_{0}\right)=0$ by a trial-and-error procedure. That is, assume a value of $\varphi_{0}$ in equation (37) and than carry out the integration. The procedure may be repeated for various values of $\varphi_{0}$ until the function in equation (37) equals zero (for computation purposes, $10^{-7}$ is set instead of zero). This is very illustrative for the students, because they see how it is possible to easily obtain the value of the incognita which is implicit in an improper integral. Figure 4 shows the results obtained for $\varphi_{0}$ as a function of $k$ when $k$ takes values between 0 and 12 . As we can see from this figure, for $k$ less than $\pi^{2} / 4, \varphi_{0}$ is zero, and if $k$ is gradually increased, there is a great increase in the angle $\varphi_{0}$. Between $k=\pi^{2} / 4=2.4674$ and $k=3, \varphi_{0}$ 
increases from 0 to approximately $\pi / 2$. On the other hand, for $k>8$, the angle $\varphi_{0}$ increases more slowly.

Knowing $\varphi_{0}$ as a function of $k$, it is easy to calculate the horizontal nondimensional tip deflection ratio using equations (23) and (26):

$$
u_{f}=\sqrt{\frac{2}{k}} \sqrt{1-\cos \varphi_{0}}
$$

and integrating equation (27) with the aid of the Mathematica program, the vertical non-dimensional tip deflection ratio can also be calculated from the following equation

$$
v_{f}=\frac{1}{\sqrt{2 k}} \int_{0}^{\varphi_{0}-\varepsilon} \frac{\cos \varphi \mathrm{d} \varphi}{\sqrt{\cos \varphi-\cos \varphi_{0}}}+\cos \varphi_{0} \sqrt{\frac{2 \varepsilon}{k \sin \varphi_{0}}}+\frac{1}{3} \sqrt{\frac{2 \varepsilon^{3} \sin \varphi_{0}}{k}}
$$

where we take $\varepsilon=10^{-12}$. Figure 5 shows the results obtained. As we can see, the values of $u_{f}$ increase rapidly between $k=\pi^{2} / 4=2.4674$ and $k=4.245$. For $k=4.245$ we obtain the maximum horizontal deflection whose value is $u_{f}=0.8056$, and from equations (20) and (30) this value is obtained when the vertical applied force is approximately $F=1.720 F_{c r}$. On the other hand, $v_{f}$ decreases when $k$ increases (for $k>$ $\left.\pi^{2} / 4\right)$. Finally, in Figure 6 the elastic curves for different values of the load parameter have been plotted.

\section{4.- Experimental results}

As an experimental example of a slender cantilever bar, the steel ruler in Figure 1 was considered. The length of the ruler is $L=30 \mathrm{~cm}$ and it has a uniform 
rectangular cross-section of width $b=3.04 \mathrm{~cm}$ and height $h=0.078 \mathrm{~cm}$. The moment of inertia of the cross section is given by the equation $I=b h^{3} / 12$, and its value is $I=$ $1.20 \times 10^{-12} \mathrm{~m}^{4}$. The weight of the ruler is $W=0.554 \mathrm{~N}$ and an external force $F$ acts on the free end of the ruler. The Young's modulus of the ruler material was calculated for small deflections and the value obtained was $E=200 \mathrm{GPa}$ [13], which implied that the flexural rigidity was $E I=0.240 \mathrm{Nm}^{2}$. Using equation (30) we can obtain the critical load for this cantilever bar, without considering its own weight, and the value obtained, $F_{c r}=6.580 \mathrm{~N}$, is much higher than $W$. We considered six applied forces whose values range from $7.448 \mathrm{~N}$ to $8.624 \mathrm{~N}$ and we measured the horizontal and vertical displacements at the free end. Figure 7 shows the results obtained as well as the results calculated with the aid of equations (36), (38) and (39) using $E I=0.240$ $\mathrm{Nm}^{2}$. It can be seen from this figure that there is good agreement between the experimental and theoretical values. Figure 8 shows the experimental elastic curve as well as the one calculated with the aid of equations (36)-(39) for an applied force $F=$ $7.644 \mathrm{~N}$.

\section{5.- Conclusions}

The post-buckling of a uniform slender cantilever bar subjected to a tip load at the free end has been theoretically, numerically and experimentally analyzed. We have shown that, although we are dealing with a simple mechanical system, it is described by a differential equation containing a non-linear term. The differential equation governing the behaviour of this system is derived without difficulty, and by analyzing this equation it is possible to show that, although straightforward in appearance, it is in fact rather difficult to solve due to the presence of a non-linear term. We have presented the solution of this differential equation in terms of elliptic 
integrals. Although the solutions to the elastica equations could be expressed in terms of elliptic functions, it is much more convenient for undergraduate students to use numerical integration as outlined in this paper. Numerical integration was performed using the Mathematica program to obtain the exact solution. This study shows that students are able to enrich their skills in maths, mechanics and computers by means of a single project. Additionally, the experiment enables students to apply wellunderstood concepts to a practical problem and highlights a number of areas in which the experiment provide students with both technical knowledge and practical evidence of the post-buckling behaviour of simple structures. We have also shown that the post-buckling of a slender cantilever bar may be easily studied with a simple, easy-toassemble, low-cost experiment, enabling the deflections of a cantilever bar to be experimentally studied by means of a series of simple measurements, such as lengths and masses. Finally, the integrated project described in this paper provides students with not only an understanding of the nonlinear post-buckling of a cantilever column but also a better understanding of the basic concepts of mechanics of materials. Important topics, including concentrated loads, linear elastic materials, modulus of elasticity, large and small deflections, moment-curvature equation, elastic curve, moments of inertia of the beam cross-section and bending moment, are considered in this experiment. 


\section{REFERENCES}

1.- L. P. Fulcher and B. F. Davis, "Theoretical and experimental study of the motion of the simple pendulum", Am. J. Phys. 44, 51-55 (1976).

2.- L. H. Cadwell and E. R. Boyko, "Linearization of the simple pendulum", Am. J. Phys. 59, 979-981 (1991).

3.- T. F. Zheng, m. Mears, D. Hall and D. Pushkin, "Teaching the nonlinear pendulum", Phys. Teach. 32, 248-251 (1994).

4.- M. I. Molina, "Simple linearizations of the simple pendulum for any amplitude", Phys. Tech. 35, 489-490 (1997).

5.- R. A. Serway, Physics for Scientific and Engineers (Saunders College Publishing, Philadelphia, 1992), p. 336.

6.- R. C. Hibbeler, Mechanics of Materials, Macmillan (1991). Chaps. 12 and 13.

7.- L. Euler, Methodus inveniendi lineas curvas maxima minimive proprietate gaudentes, Additamentum I. De Curvis Elastics (Laussane and Geneva, 1774).

8.- S. P. Timoshenko and J. M. Gere (Eds.), Theory of Elastic Stability (MacGrawHill, Snew York, 1961). Chap. 2.

9.- S. P. Timoshenko, History of Strength of Materials (New York: Dover Publications, 1983)

10.- L. D. Landau and E. M. Lifshitz, Course of Theoretical Physics, Vol. 7: Theory of Elasticity (Pergamon Press, Oxford, 1986). Chap. 17. 
11.- M. Sathyamoorthy, Nonlinear analysis of structures (CRC Press, Boca Raton FL, 1998). Chap. 1.

12.- K. Lee, "Post-buckling of uniform cantilever column under a combined load", Int. J. Non-linear Mech. 36, 813-816 (2001).

13.- A. Beléndez, C. Neipp and T. Beléndez, "Experimental study of the bending of a cantilever beam", Rev. Esp. Fis. 15 (3) $42-5$ (2001). 


\section{FIGURE CAPTIONS}

Figure 1.- Photograph of a slender cantilever bar loaded with an external vertical concentrated load at the free end.

Figure 2.- Slender cantilever bar loaded with an external vertical concentrated load at the free end.

Figure 3.- $f\left(\varphi_{0}\right)$ as a function of $\varphi_{0}$ for $k=3$.

Figure 4.- Values obtained for $\varphi_{0}$ as a function of the non-dimensional load parameter $k$.

Figure 5.- The horizontal and vertical non-dimensional tip deflection ratios, $u_{f}$ and $v_{f}$, respectively, as a function of the non-dimensional load parameter $k$.

Figure 6.- Elastic curves for different values of the load parameter $k$.

Figure 7.- Experimental and theoretical values for $u_{f}$ and $v_{f}$ as a function of the load parameter $k$.

Figure 8.- Experimental and theoretical elastic curves for the slender cantilever bar shown in Figure 1, loaded with an external vertical concentrated load $F=$ $7.644 \mathrm{~N}$ at the free end. 